\title{
Direct-Gap Gain and Optical Absorption in Germanium Correlated to the Density of Photoexcited Carriers, Doping, and Strain
}

\author{
Lee Carroll, Peter Friedli, Stefan Neuenschwander, and Hans Sigg \\ Laboratory for Micro- and Nanotechnology, Paul Scherrer Institut, CH-5232 Villigen, Switzerland \\ Stefano Cecchi, Fabio Isa, Daniel Chrastina, and Giovanni Isella \\ L-NESS, Dipartimento di Fisica del Politecnico di Milano, Polo di Como, Via Anzani 42, I-22100 Como, Italy \\ Yuriy Fedoryshyn and Jérôme Faist \\ Institute for Quantum Electronics, ETH Zurich, CH-8093 Zurich, Switzerland
}

(Received 19 January 2012; published 1 August 2012)

\begin{abstract}
Direct-gap gain up to $850 \mathrm{~cm}^{-1}$ at $0.74 \mathrm{eV}$ is measured and modeled in optically pumped Ge-on-Si layers for photoexcited carrier densities of $2.0 \times 10^{20} \mathrm{~cm}^{-3}$. The gain spectra are correlated to carrier density via plasma-frequency determinations from reflection spectra. Despite significant gain, optical amplification cannot take place, because the carriers also generate pump-induced absorption of $\approx 7000 \mathrm{~cm}^{-1}$. Parallel studies of III-V direct-gap InGaAs layers validate our spectroscopy and modeling. Our self-consistent results contradict current explanations of lasing in Ge-on-Si cavities.
\end{abstract}

DOI: 10.1103/PhysRevLett.109.057402

PACS numbers: 78.66.Db, 42.55.Px, 42.72.Ai, 78.66.Fd

Recent reports of optically pumped direct-gap lasing in tensile-strained $n$-type doped Ge [1], along with optical gain [2] in Ge microdisks, and emission from Ge photonic wires [3] and nanomembranes [4] have revitalized discussions on the feasibility of monolithically integrated laser sources compatible with Si technology. Since high performance $\mathrm{Ge}$ detectors and photomodulators already exist $[5,6]$, a monolithically grown $\mathrm{Ge}-\mathrm{on}-\mathrm{Si}$ laser could be the "missing piece" needed to realize a complementary metal oxide semiconductor compatible optoelectronics platform [7]. This platform offers the possibility of creating a highspeed high-bandwidth network to efficiently distribute data between the different multicores that exist in state-of-theart microprocessor architecture [8], without the need for the costly and difficult integration of III-V laser materials. Despite their potential to significantly impact information and communication technology, the gain and optical absorption in these materials have not been satisfactorily characterized in terms of injected carrier density or the doping and strain in the Ge layers.

To provide a solid experimental foundation for the development of a Ge laser, this Letter (i) quantifies optical gain as a function of carrier density, strain, and doping and (ii) highlights the role of valence intraband absorption in limiting optical amplification for lasing. Results from direct-gap InGaAs layers are presented as a benchmark comparison and to substantiate the spectroscopy and modeling. Our results effectively rule out the simple explanations put forward to explain current reports of lasing from doped and strained Ge-on-Si cavities [1].

As an introduction, the Bernard-Duraffourg (BD) condition states that direct-gap population inversion is obtained when the injected carrier density provides conductance-band and valence-band quasi-Fermi energy levels separated by more than the direct-gap energy $\left(E_{\mathrm{DG}}\right)$ [9]. The resulting population inversion leads to a negative differential direct-gap absorption (i.e., gain) as follows:

$$
\Delta \alpha_{\mathrm{DG}}(\hbar \omega)=\alpha_{\mathrm{DG}}(\hbar \omega)\left[1-f_{e}(\hbar \omega)-f_{h}(\hbar \omega)\right],
$$

where $f_{e}(\hbar \omega)$ and $f_{h}(\hbar \omega)$ are the quasi-Fermi-Dirac distribution probabilities of injected electrons and holes, respectively, available to participate in a vertical transition at a photon energy of $\hbar \omega$, and $\alpha_{\mathrm{DG}}(\hbar \omega)$ is the direct-gap absorption coefficient, which is proportional to the joint density of states (JDOS) and interband optical matrix elements. For typical direct-gap materials, the BD condition requires a room temperature injected carrier density of $<10^{19} \mathrm{~cm}^{-3}$ for population inversion. However, as shown in Fig. 1, for indirect-gap Ge, the energy offset between the indirect-gap energy $\left(E_{\mathrm{IG}}\right)$ and $E_{\mathrm{DG}}$ also needs to be overcome $\left(E_{\mathrm{DG}}-E_{\mathrm{IG}} \approx 140 \mathrm{meV}\right.$ for unstrained and undoped $\mathrm{Ge}$ ) [10]. As we will show, the photoexcited carrier density needed to reach inversion in $\mathrm{Ge}$ is $\approx 10^{20} \mathrm{~cm}^{-3}$, and these high densities generate proportionally strong parasitic absorption, which is sufficient to prevent the optical amplification in Ge needed for lasing, even when significant population inversion gain is achieved.

Micron thick (1-2 $\mu \mathrm{m})$ Ge layers were grown on Si by using low energy plasma enhanced chemical vapor deposition. To quantify claims of enhanced gain and emission in such films from tensile strain $\left(\varepsilon_{x x}\right)$ and $n$-type doping $\left(N_{D}\right)$, the sample series was phosphorus $n$-type doped and biaxial tensile strained by using rapid thermal annealing [11]. In this Letter, we focus on three samples that most clearly illustrate these effects: $\mathrm{Ge} 1 \quad\left(\varepsilon_{x x} \approx 0 \%\right.$ 


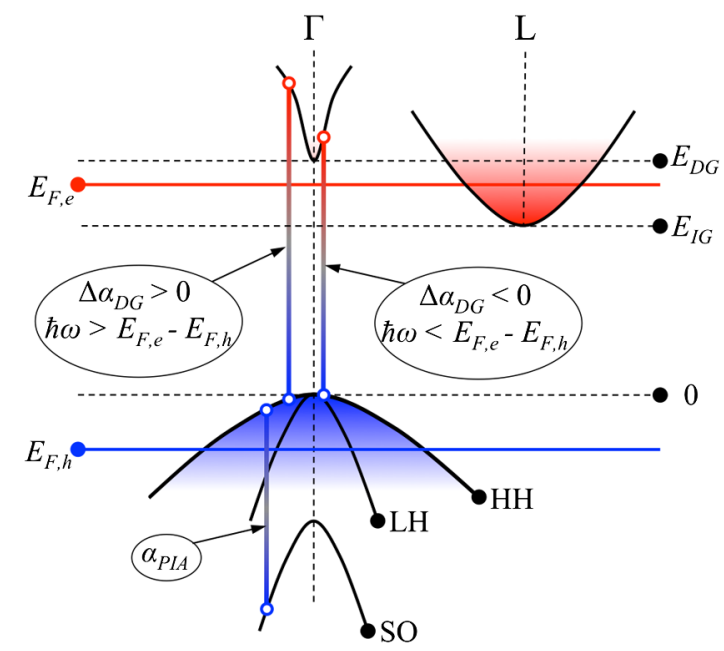

FIG. 1 (color online). Simplified band structure of unstrained $\mathrm{Ge}$, illustrating the $k$-space distribution of photoexcited carriers. $E_{F, e}$ and $E_{F, h}$ are the conductance-band and valence-band quasiFermi energy levels, respectively. The vertical HH-SO transition in the valence band thought to be responsible for most of the PIA is also indicated.

and $\left.N_{D}<10^{15} \mathrm{~cm}^{-3}\right), \operatorname{Ge} 2 \quad\left(\varepsilon_{x x} \approx 0 \%\right.$ and $N_{D}=$ $\left.2.5 \times 10^{19} \mathrm{~cm}^{-3}\right)$, and Ge3 $\left(\varepsilon_{x x}=0.25 \%\right.$ and $N_{D}<$ $10^{15} \mathrm{~cm}^{-3}$ ). Unstrained III-V direct-gap $\mathrm{In}_{0.53} \mathrm{Ga}_{0.47}$ Ason-InP samples with $n$-type $\mathrm{Si}$ doping [12] are used to benchmark the Ge-on-Si spectroscopy and modeling: InGaAs1 $\left(N_{D}<10^{15} \mathrm{~cm}^{-3}\right)$ and InGaAs2 $\left(N_{D}=\right.$ $\left.2.1 \times 10^{19} \mathrm{~cm}^{-3}\right)$.

The gain dependence on photoexcited carrier density is determined by using the infrared (IR) pump-probe system at the Swiss Light Source synchrotron, which simultaneously measures the transmission and reflection spectra under different conditions of pumping intensity and pumpprobe overlap $(\Delta t)$ with a $100 \mathrm{ps}, 1064 \mathrm{~nm} \mathrm{Nd:YAG} \mathrm{laser}$ pump pulse [13]. Examples of pumped $\mathrm{Ge}$ and InGaAs transmission spectra are given in Fig. 2(a) and 2(b), respectively. To minimize thin film interference effects, the Ge samples in Fig. 2(a) are measured in Brewster geometry. Varying $\Delta t$ gives transmission spectra corresponding to different photoexcited carrier densities. The $\Delta t=0 \mathrm{ps}$ spectrum corresponds to the maximum carrier density, while the $\Delta t=-150 \mathrm{ps}$ spectrum corresponds to a comparatively low density, given that the 100 ps probe pulse precedes the 100 ps pump pulse by 150 ps. In Fig. 2(b), the pumped InGaAs transmission clearly exceeds the normalincidence $(\mathrm{NI})$ reflection limited transmission $(\approx 60 \%)$, thereby demonstrating the existence of gain and optical amplification. This is not the case for $\mathrm{Ge}$, because the Brewster transmission remains $<100 \%$ during pumping, due to strong pump-induced absorption (PIA). As indicated in Figs. 2(a) and 2(b), and as shown more definitively by parallel mid-IR spectra, the PIA is spectrally flat for the InGaAs samples and is observed to decrease as a linear function of $\hbar \omega$ for Ge.
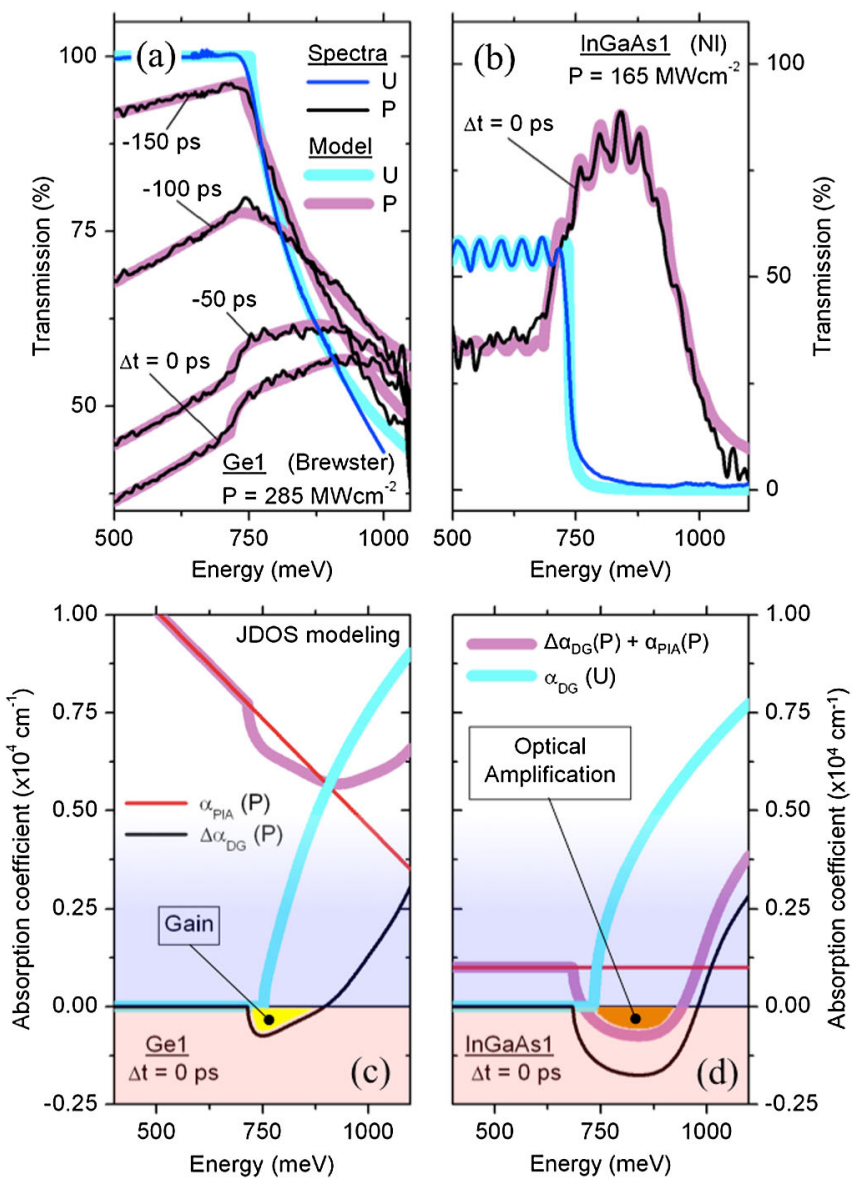

FIG. 2 (color online). (a) Brewster transmission spectra of unpumped $(U)$ and pumped $(P) \mathrm{Ge} 1$ as a function of $\Delta t$ with fits. (b) NI transmission spectra and fits of pumped InGaAs1 showing thin film interference effects and optical amplification. (c) and (d) The differential direct-gap absorption $\left(\Delta \alpha_{\mathrm{DG}}\right)$ and pump-induced absorption ( $\left.\alpha_{\text {PIA }}\right)$ extracted from the fits to spectra in (a) and (b), respectively. Optical amplification $\left(\Delta \alpha_{\mathrm{DG}}+\alpha_{\mathrm{PIA}}<0\right)$ and gain $\left(\Delta \alpha_{\mathrm{DG}}<0\right)$ ranges are highlighted.

The transmission spectra in Figs. 2(a) and 2(b) are fitted with a model to determine $\Delta \alpha_{\text {DG }}$ from Eq. (1). The model accounts for reflection losses and interference effects in the thin lamella and models $\alpha_{\mathrm{DG}}(\hbar \omega)=A^{*} \sqrt{\hbar \omega-E_{\mathrm{DG}}}$, with the light-hole (LH) and heavy-hole (HH) absorption contributions added separately [9]. The quasi-Fermi-Dirac statistics in the JDOS model use a sample temperature of $\approx 400 \mathrm{~K}$ as deduced from "unpumped" probe-pulse spectra of the absorption edge of the samples [14]. Using existing or independently determined (see discussions below) effective carrier masses, this model is fitted to the spectra, varying the injected carrier density as the only fitting parameter. Once the flat or linear PIA contribution is introduced, this model accurately fits the pumped and unpumped Ge and InGaAs spectra in Figs. 2(a) and 2(b). The values of $\Delta \alpha_{\mathrm{DG}}(\hbar \omega)$ and PIA absorption coefficient $\alpha_{\text {PIA }}(\hbar \omega)$ from these fits are shown in Figs. 2(c) and 2(d). The reason why InGaAs provides optical amplification and 
Ge does not is clear: Although pumped Ge1 has a significant direct-gap gain of $850 \pm 50 \mathrm{~cm}^{-1}$, the corresponding $\alpha_{\mathrm{PIA}}\left(\hbar \omega=E_{\mathrm{DG}}\right) \quad$ is $\approx 7000 \mathrm{~cm}^{-1}$. Since $\Delta \alpha_{\mathrm{DG}}+$ $\alpha_{\text {PIA }}\left(E_{\text {DG }}\right)>0$, no amplification can occur. In contrast, InGaAs1 has a peak gain of $1750 \pm 50 \mathrm{~cm}^{-1}$ with $\alpha_{\text {PIA }}\left(E_{\mathrm{DG}}\right)=1000 \pm 50 \mathrm{~cm}^{-1}$, providing strong optical amplification over a spectral bandwidth of $\approx 200 \mathrm{meV}$. For both high- and low-level optical excitation, the results from all Ge samples are the same-no optical amplification is observed within the $\pm 50 \mathrm{~cm}^{-1}$ limits of experimental accuracy. This is consistent with direct-gap photoluminescence spectra from the samples, which show no evidence of stimulated emission that would point to optical amplification.

In order to provide a quantitative basis for the development of Ge-on-Si laser devices, these gains and PIA losses must be correlated to the carrier density (for different conditions of strain and doping). This correlation is made through an analysis of the carrier plasma frequency $\left(\omega_{P}\right)$, which introduces a minimum in the mid-IR NI reflection spectrum close to $E_{P}=\hbar \omega_{p}=\hbar \sqrt{N_{T} e^{2} / m_{P} \varepsilon}$, where $m_{P}$ is the plasma mass, $e$ is the elementary charge, and $N_{T}$ is the total carrier density [15]. The total carrier density $N_{T}$ is the sum of the photoexcited carriers $\left(N_{P}\right)$ and dopant density $\left(N_{D}\right)$. Figure 3(a) shows examples of measured mid-IR reflection spectra [expressed in terms of the ratio of pumped $\left(R_{P}\right)$ and unpumped $\left(R_{U}\right)$ reflection spectra] fitted with a Drude model whose free parameters are the plasma frequency of the (known) $N_{D}$ and unknown $N_{P}$ [15]. These two fitting parameters allow the effective mass of the electrons to be deduced (and determined as a function of $N_{D}$ from the doping series [16] to reveal nonparabolicity effects [17]) and combined with existing LH and $\mathrm{HH}$ masses to calculate the $m_{P}$ of the carriers $[18,19]$. The inset in Fig. 3(a) shows the extracted values of $N_{P}$ in Ge1 as a function of pumping intensity using this approach. The plasma mass is $m_{P}=\left(m_{e}^{-1}+m_{h}^{-1}\right)^{-1}=0.08 m_{0}$, where $m_{0}$ is the vacuum electron mass, $m_{e}=0.12 m_{0}$ is the $L$-valley $\mathrm{Ge}$ electron effective mass of conductivity (not the effective mass of the density of states), and $m_{h}=$ $0.25 m_{0}$ calculated from an equilibrium filling of the $\mathrm{HH}$ and LH bands $\left(m_{\mathrm{HH}}=0.33 m_{0}\right.$ and $\left.m_{\mathrm{LH}}=0.043 m_{0}\right)$ [18]. The nonlinearity between carrier density and pumping intensity in Fig. 3(a) is due to Auger recombination, as demonstrated by fitting the data with an Auger model that uses a recombination time of $\tau_{R}^{-1}=\gamma N_{P}^{2}$, to return a value of $\gamma=3.2 \pm 0.3 \times 10^{-30} \mathrm{~cm}^{6} \mathrm{~s}^{-1}$, which is within a factor of $\times 2$ of existing values [20]. Figure 3(b) compares the carrier density values determined from fitting to the transmission JDOS model and from fitting to the Drude model. The excellent correspondence between the two approaches is a strong validation of the spectroscopy and modeling. Note that, while the Auger-limited recombination time during pumping of the Ge samples is $\approx 30 \mathrm{ps}$, this quickly stabilizes to $\tau_{R}=3.3 \pm 0.5 \mathrm{~ns}$ after pumping.
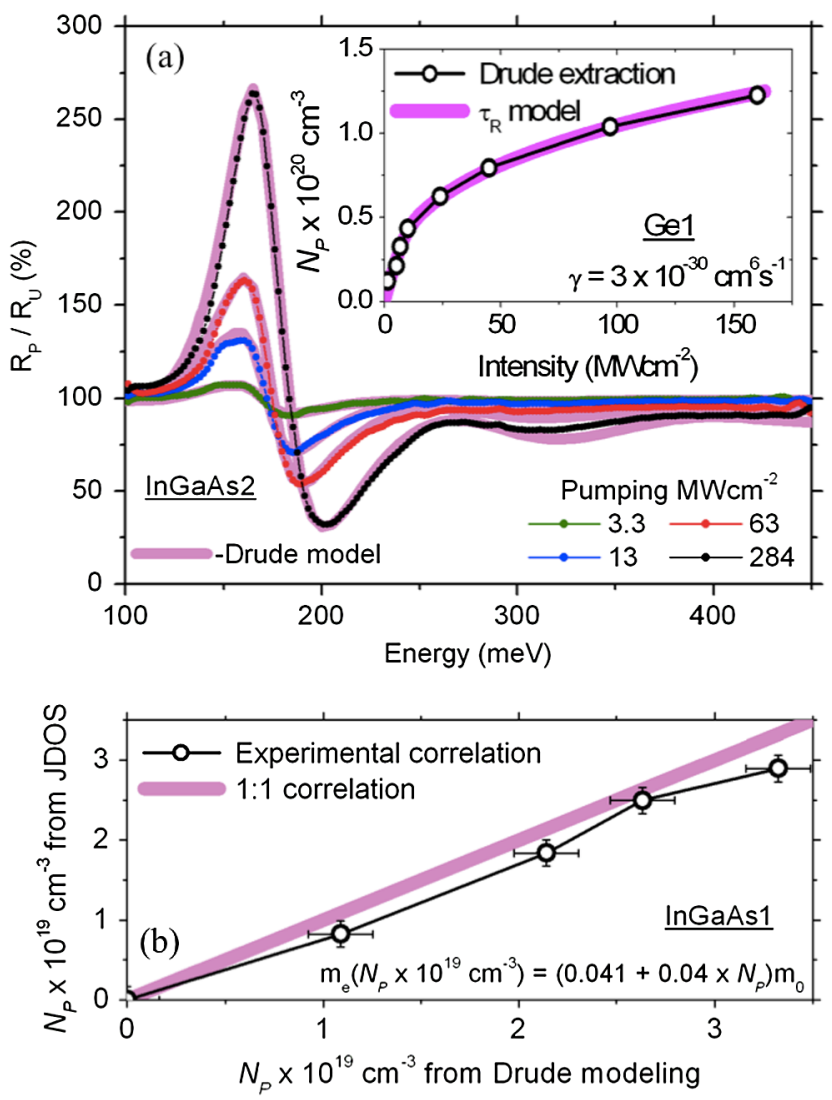

FIG. 3 (color online). (a) Mid-IR reflection spectra of pumped InGaAs 2 expressed as the ratio of pumped $\left(R_{P}\right)$ and unpumped $\left(R_{U}\right)$ reflection, fitted with a Drude model. The inset shows the deduced injected carrier density in $\mathrm{Ge} 1$ as a function of pumping intensity. (b) Near unity correlation between injected carrier density values for InGaAs1 as deduced independently from Drude modeling and transmission modeling. The observed variation in electron effective mass in InGaAs [16] from conductanceband nonparabolicity has been included in these calculations.

This implies a radiative recombination parameter of $\approx 10^{-10} \mathrm{~cm}^{3} \mathrm{~s}^{-1}$, which is a typical value for direct-gap materials, and shows that significant direct-gap recombination takes place in the photoexcited $\mathrm{Ge}$.

Figure 4 summarizes the two critical optical parameters needed to design or model a Ge-on-Si laser element-the direct-gap gain and the PIA as a function of injected carrier density (for different strain and doping). Note that for the Brewster-geometry data in Fig. 2(a), the corresponding reflection spectra do not provide a convenient relation to the injected carrier density. For these spectra, the density corresponding to the gain value is found by correlating the Brewster-geometry determination of $\alpha_{\mathrm{PIA}}\left(E_{\mathrm{DG}}\right)$ to the values in Fig. 4(b), which were made at NI. The high degree of linearity allows for confident extrapolation and interpolation of these $\alpha_{\mathrm{PIA}}\left(E_{\mathrm{DG}}\right)$ as a function of $N_{T}$.

In Fig. 4(a), the measured gain is compared to predictions of the JDOS model from Figs. 2(c) and 2(d). The injected density for the onset of gain $\left(\Delta \alpha_{\mathrm{DG}} \leq 0\right)$ is 


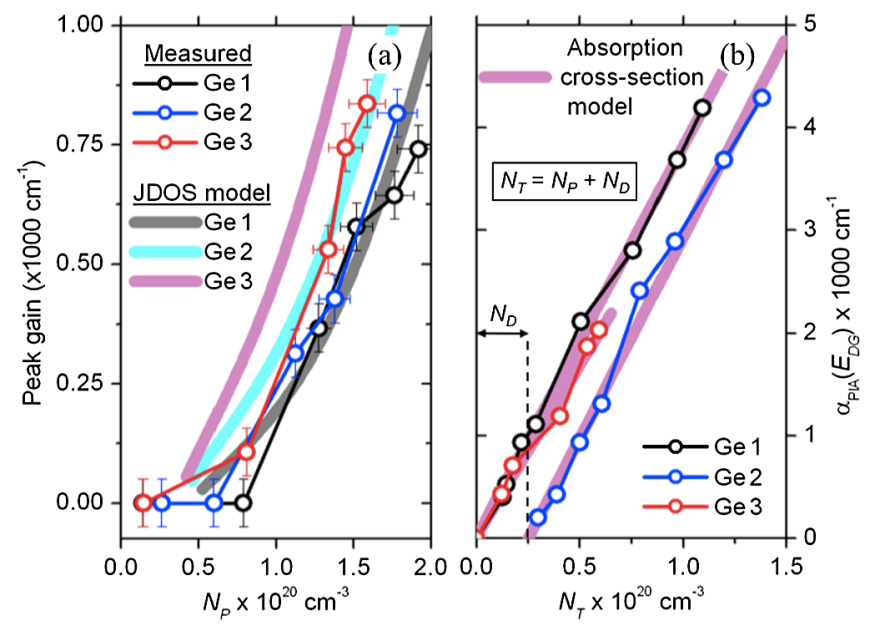

FIG. 4 (color online). (a) Comparison of measured and modeled peak gain values $\left(-\Delta \alpha_{\mathrm{DG}}\right)$ from the Ge samples as a function of carrier injection. (b) The magnitude of the PIA at the direct-gap energy, for the three Ge samples, expressed as a function of total carrier density, fitted by using a linear crosssection model. Note that $\Delta \alpha_{\mathrm{DG}}+\alpha_{\mathrm{PIA}}\left(E_{\mathrm{DG}}\right) \gg 0$ in all cases, meaning that optical amplification cannot occur.

$7 \pm 1 \times 10^{19} \mathrm{~cm}^{-3}$ for $\mathrm{Ge} 1$ (undoped and unstrained) and lower for the doped and strained samples $(\mathrm{Ge} 2$ : $5 \pm 1 \times 10^{19} \mathrm{~cm}^{-3}$ and Ge3: $\left.6 \pm 1 \times 10^{19} \mathrm{~cm}^{-3}\right)$. The $n$-type doping and tensile strain reduce the onset by making the BD inversion condition easier to satisfyincreasing the quasi-Fermi level of the conduction band and reducing the energy offset between $E_{\mathrm{DG}}$ and $E_{\mathrm{IG}}$, respectively. There is a fair agreement between the measured gain and JDOS predictions. The lower onset values predicted by the model than those observed are likely due to nonparabolicity effects, analogous to those observed in the InGaAs [16] (but activated for higher densities in the case of $\mathrm{Ge}$ ), and the band-gap renormalization from carrier injection that more strongly reduces the $L$-valley gap than the $\Gamma$-valley gap [21]. While the $\Gamma$-valley band-gap renormalization can be extracted from Fig. 2(c) and is $-42 \pm$ $3 \mathrm{meV}$ for a carrier density of $1.9 \pm 0.2 \times 10^{20} \mathrm{~cm}^{-3}$, our transmission analysis cannot probe the $L$-valley renormalization, because the indirect absorption cross section is too low. Both nonparabolicity and differential renormalization between the $L$ and $\Gamma$ valleys are currently outside our modeling but are known to make the BD condition more difficult to satisfy and thereby raise the gain onset.

As highlighted earlier, despite a significant gain of up to $850 \pm 50 \mathrm{~cm}^{-1}$ in the Ge samples, there is no optical amplification, because of strong PIA. Figure 4(b) gives $\alpha_{\mathrm{PIA}}\left(\hbar \omega=E_{\mathrm{DG}}\right)$ as a function of the carrier density for the three Ge samples (results from the InGaAs samples are completely analogous). The PIA data are accurately fit by a linear cross-section model that accounts for the electron $\left(\sigma_{e}\right)$ and holes $\left(\sigma_{h}\right)$ separately: $\alpha_{\mathrm{PIA}}\left(E_{\mathrm{DG}}\right)=\sigma_{e} N_{e}+$ $\sigma_{h} N_{h}$. For undoped samples, the electron density $\left(N_{e}\right)$ and hole density $\left(N_{h}\right)$ are equal to the photoexcited carrier density of $N_{P}$. For $n$-type doped samples, $N_{e}$ is given by $N_{T}=N_{P}+N_{D}$, where $N_{T}$ is the total carrier density, defined as the sum of photoexcited and dopant densities. The doping introduces an offset of $N_{D}$ in the data of Ge2 plotted in Fig. 4(b) but does not appreciably change the absorption cross section. Fitting to these data gives $\sigma_{h}=$ $3.8 \pm 0.2 \times 10^{-3} \mathrm{~nm}^{2}$ and $\sigma_{h} / \sigma_{e}=12 \pm 1$, in good agreement with existing $n$-type [22] and $p$-type [23] doped absorption data (giving $\sigma_{h} \approx 5 \times 10^{-3} \mathrm{~nm}^{2}$ and $\sigma_{h} / \sigma_{e} \approx 10$ ). Note that $\sigma_{h} \gg \sigma_{e}$, because the holes may undergo a vertical transition, leading to valence intraband absorption, for which there is no conduction band analogue at $\hbar \omega \approx E_{\mathrm{DG}}$ [23]. As illustrated in Fig. 1, the HH-SO transition is likely the dominant source of valence intraband absorption near $E_{\mathrm{DG}}$, because of the energy offset and the relative $\mathrm{HH}$ and SO masses. Note that valence intraband absorption has not been properly accounted for in existing modeling of gain in Ge [10], and this has led to a significant underestimation of the challenges in achieving optical amplification and provided an erroneous framework in which reports of Ge-on-Si lasing [1] have been interpreted.

From Fig. 4 , it is clear that $\Delta \alpha_{\mathrm{DG}}+\alpha_{\mathrm{PIA}}\left(E_{\mathrm{DG}}\right) \gg 0$ for all samples at all injected carrier densities, even those with high doping and biaxial tensile strain. For injected densities of $\approx 10^{20} \mathrm{~cm}^{-3}$, the PIA is typically $\times 20$ higher than the gain, demonstrating that optical amplification is not even marginally probable. Note that the PIA is already $\approx 500 \mathrm{~cm}^{-1}$ for the predicted gain onset densities, and such results clearly go against the current explanations of lasing from Ge-on-Si cavities [1]. While the injected density in these waveguide cavities is not known, it can be estimated by applying the pumping conditions to the Auger model used to fit the inset in Fig. 3(b). This approach estimates an injection density of $N_{P}=1 \times 10^{19} \mathrm{~cm}^{-3}$ for the cavities, which is a factor of $\approx 3$ below the JDOS model prediction for their gain onset (given the reported cavity strain and doping levels). Even if the cavity structure provides significantly better carrier confinement and lower defect densities than the Ge-on-Si films, such that injected density is actually $3.5 \times 10^{19} \mathrm{~cm}^{-3}$, the resulting gain is only $\approx 80 \mathrm{~cm}^{-1}$ with $\alpha_{\mathrm{PIA}}\left(E_{\mathrm{DG}}\right) \approx 500 \mathrm{~cm}^{-1}$. Clearly, light amplification under such conditions is impossible, and it remains to be understood how the reported emission spectra in Ref. [1] can contain Fabry-Pérot oscillations and other modal structure that indicate at least optical transparency [defined as $\Delta \alpha_{\mathrm{DG}}+\alpha_{\mathrm{PIA}}\left(E_{\mathrm{DG}}\right)=0$ ], when such a condition is so clearly impossible for the homogenous $2 \mathrm{D} \mathrm{Ge}-\mathrm{on}-\mathrm{Si}$ layers. One tentative explanation is that the inhomogeneous strain distribution in the selectively grown micron-sized Ge waveguide cavities is playing a role.

In summary, photoexcited carriers generate considerable direct-gap gain in Ge-on-Si layers but also strong PIA that prohibits optical amplification. Our spectroscopy offers a means of correlating the key optical parameters 
of direct-gap gain and PIA to injected carrier density and shows that recent reports of lasing from Ge-on-Si cavities cannot be explained in terms of the optical parameters of homogenous Ge layers.

We thank Dr. Philippe Lerch and Dr. Luca Quaroni of the Swiss Light Source X01DC beam line for their technical assistance and Stefan Stutz for sample preparation. This work was partially funded by the Swiss National Fund and the CARIPLO foundation NanoGap project.

[1] J. Liu, X. Sun, R. Camacho-Aguilera, L. C. Kimerling, and J. Michel, Opt. Lett. 35, 679 (2010).

[2] J. Liu, X. Sun, D. Pan, X. Wang, L. C. Kimerling, T. L. Koch, and J. Michel, Opt. Express 15, 11272 (2007).

[3] M. de Kersauson, M. El Kurdi, S. David, X. Checoury, G. Fishman, S. Sauvage, R. Jakomin, G. Beaudoin, I. Sagnes, and P. Boucaud, Opt. Express 19, 17925 (2011).

[4] J. R. Sánchez-Péreza, C. Boztug, F. Chen, F. F. Sudradjat, D. M. Paskiewicz, R. B. Jacobson, M. G. Lagally, and R. Paiella, Proc. Natl. Acad. Sci. U.S.A. 108, 18893 (2011).

[5] J. Michel, J. Liu, and L. C. Kimerling, Nature Photon. 4, 527 (2010).

[6] G. T. Reed, G. Mashanovich, F. Y. Gardes, and D. J. Thomson, Nature Photon. 4, 518 (2010).

[7] D. Liang and J. E. Bowers, Nature Photon. 4, 511 (2010).

[8] M. J. Paniccia, Opt. Photonik 6, 34 (2011).

[9] J.I. Pankove, Optical Processes in Semiconductors (Dover, New York, 1975).
[10] J. Liu, X. Sun, D. Pan, X. Wang, L. C. Kimerling, T.L. Koch, and J. Michel, Opt. Express 15, 11272 (2007).

[11] L. Carroll, F. Imbert, H. Sigg, M. Süess, E. Müller, M. Virgilio, G. Pizzi, P. Rossbach, D. Chrastina, and G. Isella, Appl. Phys. Lett. 99, 031907 (2011).

[12] Y. Fedoryshyn, M. Beck, P. Kaspar, and H. Jaeckel, J. Appl. Phys. 107, 093710 (2010).

[13] L. Carroll, P. Friedli, P. Lerch, J. Schneider, D. Treyer, S. Hunziker, S. Stutz, and H. Sigg, Rev. Sci. Instrum. 82, 063101 (2011).

[14] E. Zielinski, H. Schweizer, K. Streubel, H. Eisele, and G. Weimann, J. Appl. Phys. 59, 2196 (1986).

[15] F. Stern, Solid State Phys. 15, 299 (1963).

[16] Our analysis of doped InGaAs samples indicates that the optical mass of the electrons is linearly proportional to the doping density: $m_{e}\left(N_{D} \times 10^{19} \mathrm{~cm}^{-3}\right)=$ $\left(0.04+0.04 \times N_{D}\right) \times m_{0}$, where $m_{0}$ is the electron vacuum mass.

[17] C. Skierbiszewski, S. P. Lepkowski, P. Perlin, T. Suski, W. Jantsch, and J. Geisz, Physica (Amsterdam) 13E, 1078 (2002).

[18] S. Sze, Physics of Semiconductor Devices (Wiley, New York, 1981), 2nd ed.

[19] W. G. Spitzer and H. Y. Fan, Phys. Rev. 106, 882 (1957).

[20] N. G. Nilsson, Phys. Scr. 8, 165 (1973).

[21] J. I. Pankove and P. Aigrain, Phys. Rev. 126, 956 (1962).

[22] W. G. Spitzer, F. A. Trumbore, and R. A. Logan, J. Appl. Phys. 32, 1822 (1961).

[23] R. Newman and W.W. Tyler, Phys. Rev. 105, 885 (1957). 